EPJ Web of Conferences 23, 00017 (2012)

DOI: $10.1051 /$ epjconf/20122300017

(C) Owned by the authors, published by EDP Sciences, 2012

\title{
Graphene edges; localized edge state and electron wave interference
}

\author{
Toshiaki Enoki \\ Tokyo Institute of Technology, Department of Chemistry, Ookayama, Meguro-ku, Tokyo 152-8551, Japan
}

\begin{abstract}
The electronic structure of massless Dirac fermion in the graphene hexagonal bipartite is seriously modified by the presence of edges depending on the edge chirality. In the zigzag edge, strongly spin polarized nonbonding edge state is created as a consequence of broken symmetry of pseudo-spin. In the scattering at armchair edges, the K-K' intervalley transition gives rise to electron wave interference. The presence of edge state in zigzag edges is observed in ultra-high vacuum STM/STS observations. The electron wave interference phenomenon in the armchair edge is observed in the Raman G-band and the honeycomb superlattice pattern with its fine structure in STM images.
\end{abstract}

\section{Introduction; the role of edges in the electronic structure}

Recently massless Dirac fermions have been paid great attention in condensed matter physics [1-4]. Among the materials, in which the electronic features of massless Dirac fermion are observed, graphene, single sheet of graphite, is of particular importance [2-4]. The successful isolation of graphene and the discovery of its unconventional electronic properties, with which 2010 Nobel Prize in Physics were award to A. Geim and K. Novoselov, have made it popular for condensed matter physicists [2].

The electronic structure of graphene is described in terms of Weyl equation in relativistic quantum mechanics as given in the following equation;

$$
\hat{H}=v_{\mathrm{F}} \sigma \mathbf{P} \text {, }
$$

where $v_{\mathrm{F}} \sigma$ and $\mathbf{P}$ are the Fermi velocity, pseudo-spin and the momentum, respectively. The linear momentum dependence of the kinetic energy gives rise to the creation of cone-shaped $\pi$-valence and $\pi^{*}$-conduction bands (Dirac cones), the top of the former and the bottom of the latter touching to each other at the Dirac point. What is important here is that the fermion is moving on the hexagonal bipartite lattice of a graphene sheet consisting of two independent sublattices A and B as given in Fig.1(a). The structural degree of freedom 2 in the bipartite lattice accordingly gives two independent Dirac points $\mathrm{K}$ and $\mathrm{K}^{\prime}$ at the Brillouin zone edges as exhibited in Fig.2. In addition to this, the double degeneracy is effectively converted to the pseudo-spin $\sigma$ in Weyl equation.

When graphene is cut into fragments and the created edges are terminated with hydrogen atoms, we can obtain semi-infinite graphene, nanographene, to small condensed polycyclic aromatic hydrocarbon molecules. Here there are two independent cutting directions; zigzag and armchair directions. The edges in these graphene fragments play an important role in modifying the electronic structure of graphene sheet depending on their edge chirality in the bipartite lattice as summarized in the following two issues $[5,6]$. One is the creation of edge state localized in zigzag edges [7-12], while the other is the electron wave interference in armchair edges $[6,13]$. The creation of edge state is understood as a consequence (a)

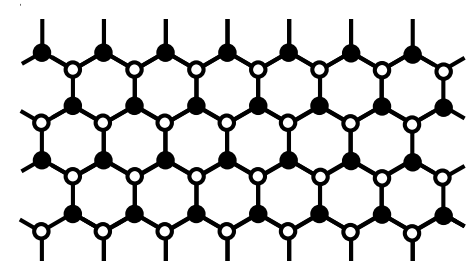

(b)

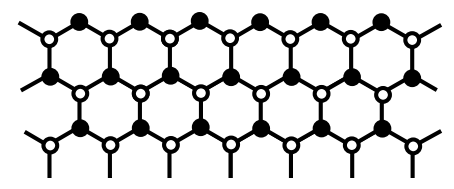

(c)

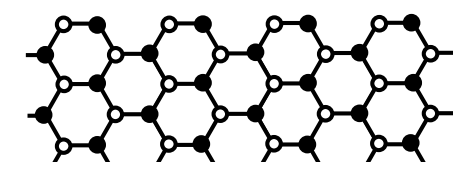

Fig. 1. (a) Hexagonal bipartite lattice consisting of two independent sublattices; A (filled circle) and B (open circle) in graphene. (b) Zigzag edge. (c) Armchair edge.

This is an Open Access article distributed under the terms of the Creative Commons Attribution-Noncommercial License 3.0, which permits unrestricted use, distribution, and reproduction in any noncommercial medium, provided the original work is properly cited 
(a)

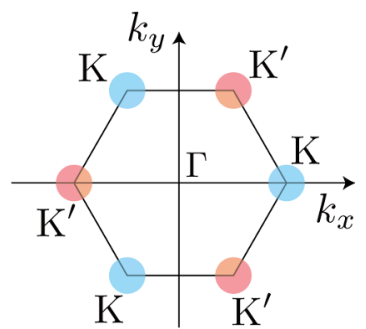

(b)

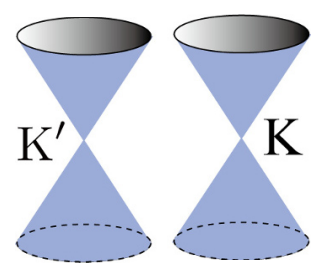

(a)

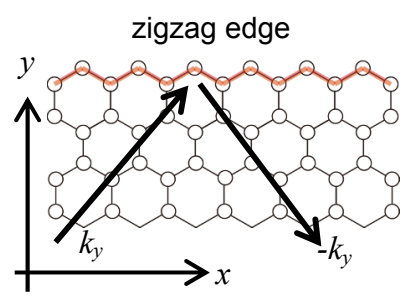

(b) armchair edge

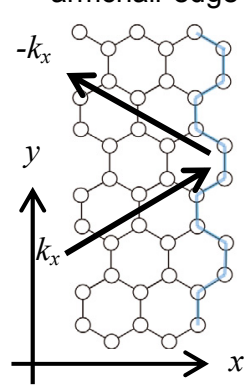

Fig. 2. Brillouin zone of graphene (a) and the Dirac cones at $\mathrm{K}$ and $\mathrm{K}$ ' points.

of the broken symmetry in the pseudo-spin of the Dirac fermion at zigzag edges. Indeed, zigzag edge has only one of the sublattices, whereas A and B sublattices are always paired in armchair edge, as illustrated in Fig.1(a) and (b). The electron wave interference is related to the presence of two Dirac cones at $\mathrm{K}$ and $\mathrm{K}$ ' points as shown in Fig.2. In the scattering event at a zigzag edge shown in Fig.3(a), in which $k_{y}$ is converted to $-k_{y}$, only one Dirac cone $(\mathrm{K}$ or $\mathrm{K}$ ') participates as the intravalley transition

(a)
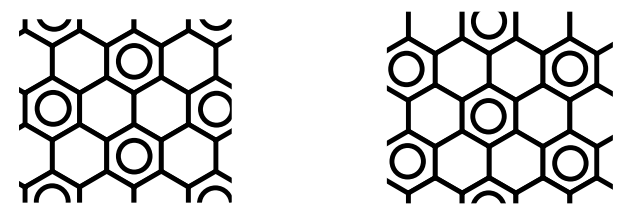

(b)

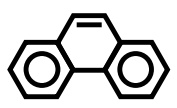

(2)

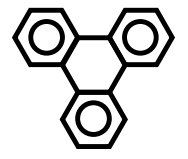

(3)

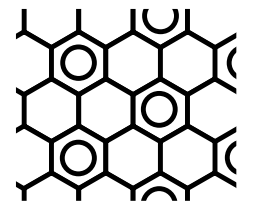

Fig. 3. Scattering of an electron in a zigzag edge (a) and an armchair edge (b).

with no interference, whereas the intervalley transition between $\mathrm{K}$ and $\mathrm{K}$ ' features the scattering process in an armchair edge (Fig.3(b)), in which $k_{x}$ is converted to $-k_{x}$, giving interference between $\mathrm{K}$ and $\mathrm{K}^{\prime}$ points as given in the following equation for a site at $\mathbf{R}_{B}$ of sublattice $\mathrm{B}$,

$$
\psi_{B}\left(\mathbf{R}_{B}\right) \propto\left(-\exp \left(-i \mathbf{K} \mathbf{R}_{B}\right)+\exp \left(i \mathbf{K}^{\prime} \mathbf{R}_{B}\right)\right) .
$$

Here let us discuss the issues on the edge state and interference in chemistry language since the graphene fragments are involved in the family of polycyclic

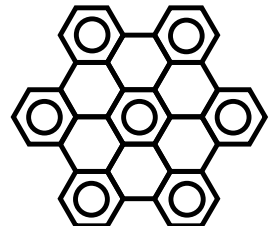

(7)

(c)

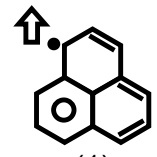

(1)

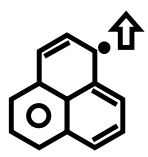

(1)

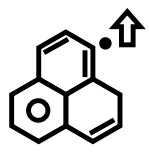

(1)

(d)

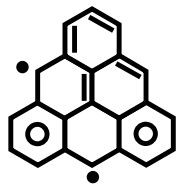

(2)

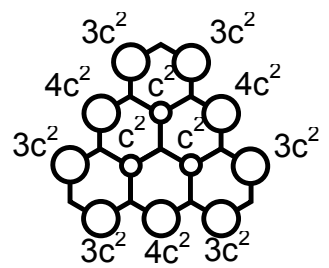

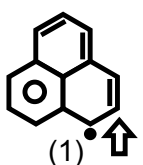

$S=1 / 2[1]$

Fig. 4. Clar's representations. (a) Infinite graphene. Three states are degenerate with each state forming a $\sqrt{3} \times \sqrt{3}$ superlattice. (b) Armchair-edged molecules. (c) Zigzag-edged phenalenyl radical having 12 degenerate states. Here only three of them are drawn due to three fold symmetry of the molecule. (d) Zigzag-edged triangulene and its spatial distribution of the edge state (right). The number in the parenthesis denotes the number of constituent sextets, and that in the bracket is the number of unpaired electrons, which corresponds to the number of edge states. The arrow and $\mathrm{S}$ are edge-state spin and its quantum state. 
aromatic hydrocarbon molecules. Chemists should remind an important phenomenological rule called Clar's aromatic sextet rule in discussing the electronic structure and chemical activities of aromatic molecules [14-16]. The aromatic sextet (expressed by a hexagon with a circle in its center), which is the fundamental unit in Clar's theory, is a benzene molecule, which is given as Kekule resonance state and is bonded to the surrounding with six single bonds. According to the Clar's rule, the electronic structure and thermodynamical stability can be understood by tiling aromatic sextets on a molecule concerned. Here the most stable state is given with the structure having a maximal number of sextets.

In infinite size graphene, aromatic sextets are placed in every three hexagon rings. Accordingly, three states given by the Clar's representation are degenerate, forming a $\sqrt{3} \times \sqrt{3}$ superstructure, as shown in Fig.4(a). This means that infinite graphene has aromaticity weaker $1 / 3$ than benzene, giving electronic and chemical acivity stronger than benzene. In the meantime, armchair-edged molecules, a large number of sexets can be placed as revealed in Fig.4(b). Therefore armchair-edged molecules are thermodynnamically stable. Interestingly and importantly, these armchair-edged molecules can be described in terms of only one unique state, suggesting the presence of a standing wave. This is a consequence of electron wave interference as already discussed from physics side. In contrast, there are many Clar's representations degenerate in zigzag-edged molecules as given in Fig.4(c) and (d). For example, phenalene (phenalenyl radical) has 12 degenerate states with the absence of standing wave. In addtion, zigzag-edged molecules have a small number of sextets, being proved to be electronically and chemically active with the presence of nonbonding $\pi$-electron states. In the radical representation, zigzag-edged phenalene and trigangulene consising of 3 and 6 hexagon rings fused in triangle shape, respectively, have 1 and 2 nonbonding states which are magnetic with $S=1 / 2$ and 1, respectively, owing to Hund rule. Here the spin state is described in terms of Lieb's theorem;

$$
S=(1 / 2)\left|N^{A}-N^{B}\right|
$$

where $N^{A}$ and $N^{B}$ are the numbers of carbon sites belonging to sublattices $\mathrm{A}$ and $\mathrm{B}$, respectively. Figure 4(d) reveals that the nonbonding $\pi$-electron state is populated in the zigzag edge region. This is the reason why the nonbonding state is called edge state. Note that armchair-edge molecules are in the closed shell electronic state with no unpaired electrons.

In the next two sections, let us discuss the localized edge state and electron wave interference on the basis of experimental observations.

\section{Edge state in zigzag edges}

It is particularly important to show the evidence on the presence of edge state using scanning tunneling microscopy/spectroscopy (STM/STS) technique in atomic resolution [10-12]. However, graphene edges prepared in the ambient atmospheric condition are usually covered heavily with oxygen-containing functional groups due to the easy oxidation of graphene edges in the ambient condition [18]. STM observations with hydrogen-terminated graphene edges are required to examine the electronic structure of the graphene edges in detail. For this reason, the graphene edges are heattreated at $800{ }^{\circ} \mathrm{C}$ to remove the oxygen-containing functional groups and hydrogen terminated in ultra-high vacuum (UHV) condition [10,11]. Figure 5 exhibits the typical examples of the UHV-STM lattice images and STS spectra of graphene edges which are hydrogen terminated [10]. Figures 5(a) and (b) are the STM and STS results for a uniform armchair edge, respectively. The STS spectrum demonstrates that the electronic structure in the vicinity of the Fermi level $\left(E_{\mathrm{F}}\right)$ is the same to that of infinite graphene sheet with a feature of massless Dirac fermion described in terms of linear valence $\pi$ - and conduction $\pi^{*}$-bands which touch to each other at $E_{\mathrm{F}}$ [4]. This reflects the lattice image which shows a $\sqrt{3} \times \sqrt{3}$ superlattice structure with no additional contribution. Armchair edges observed are generally long and less defective, being suggested to be energetically stable, in accordance with the aromatic stability predicted by theory [14-16]. Zigzag edges have features that are different from armchair edges. Zigzag edges tend to be defective and short. They are observed frequently to be embedded between armchair edges, as shown in Fig.5(c). This is a consequence of the energetically unstable structure of less aromatic zigzag edges [14-16]. What is important in zigzag edges is the presence of edge state [79]. The STS spectrum (Fig.5(d)) at the zigzag edge shows a sharp peak of the density-of-states at $E_{\mathrm{F}}$ in addition to the linear $\pi$ - and $\pi^{*}$-bands, giving evidence for the edge state. The bright spots observed in the zigzag edge region (Fig.5(c)) are ascribed to the edge state. (a)

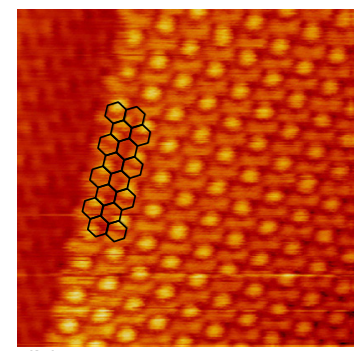

(b)

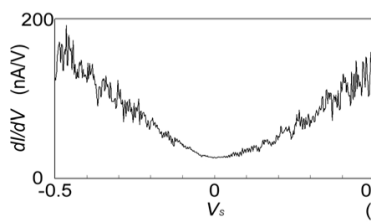

(c)

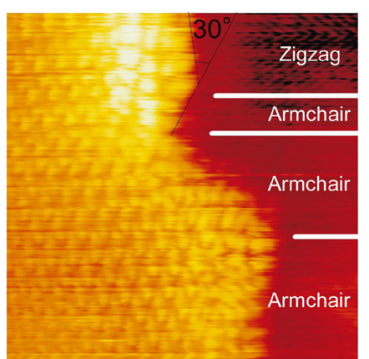

(d)
Fig. 5. (a) Atomically resolved UHV STM image $(5.6 \times 5.6$ $\mathrm{nm}^{2}$ ) of a homogeneous armchair edge. To clarify the edge structures, a model of the honeycomb lattice is overlaid on the image. (b) $\mathrm{d} I / \mathrm{d} V_{\mathrm{s}}$ curve from STS measurements taken at the edge in (a). (c) Atomically resolved UHV STM image of zigzag and armchair edges $\left(9 \times 9 \mathrm{~nm}^{2}\right)$. (d) $\mathrm{d} I / \mathrm{d} V_{\mathrm{s}}$ curve from STS data at a zigzag edge in (c). Images were taken in constant-height mode with a bias voltage $V_{\mathrm{s}}=0.02 \mathrm{~V}$ and current $I=0.7 \mathrm{nA}$. [Ref.10] 


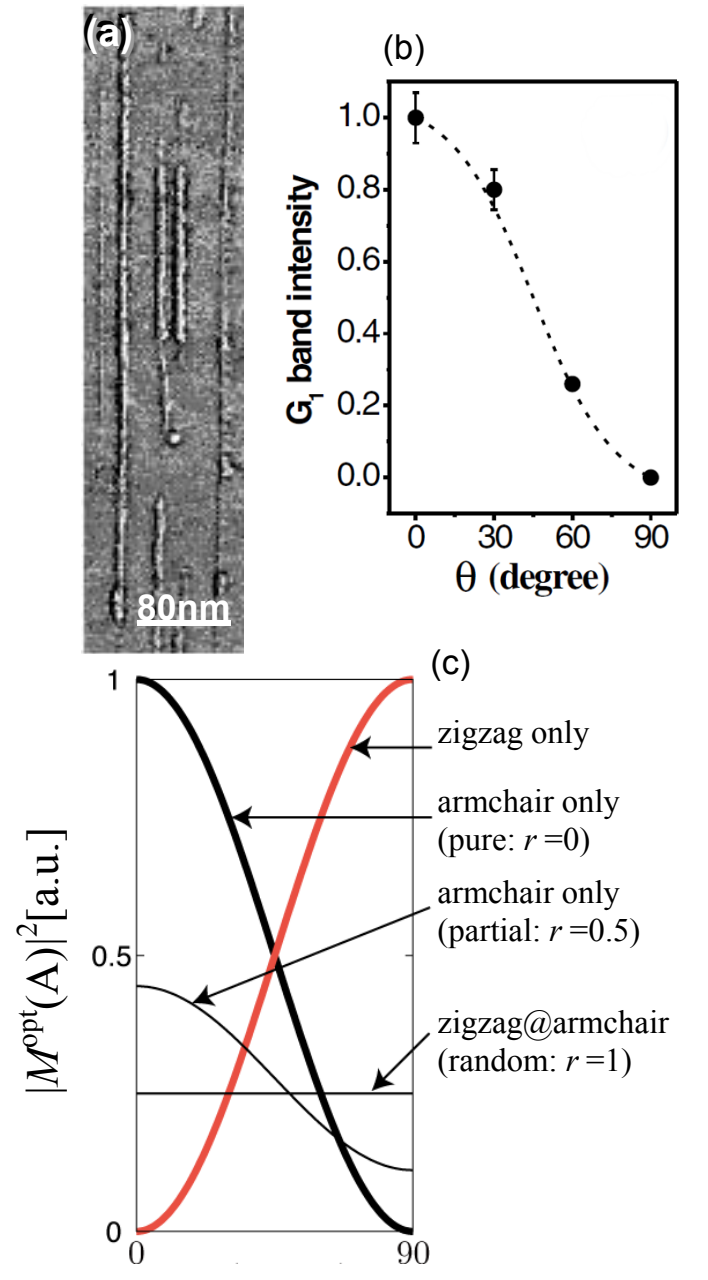

Fig. 6. (a) AFM image of armchair-edged single layer graphene nanoribbons prepared by heat-treatment of a graphite step edge at $1600{ }^{\circ} \mathrm{C}$. The typical size is $8 \mathrm{~nm}$ in width and 1 $\mu \mathrm{m}$ in length. (b) Experimentally observed laser beam polarization angle dependence of the Raman G-band intensity of the graphene nanoribbon shown in (a), where $\theta$ is the angle between ribbon direction and the polarization direction of the incident laser beam. [Ref.21] (c) Theoretical polarization angle dependence of the G-band intensity for zigzag, armchair and zigzag/armchair mixed edges. [Ref.6]

\section{Electron wave interference at armchair edges}

\subsection{Raman G-band}

The graphite $E_{2 \mathrm{~g} 2}$ mode ( $\mathrm{G}$ band) observed around 1600 $\mathrm{cm}^{-1}$ in the resonance Raman spectrum is an important signature indicating graphitic structure [19]. The lattice vibration modulates the inter-atomic $\mathrm{C}-\mathrm{C}$ distance, distorting the three-fold symmetric bond coordination surrounding the carbon atom. This breaks the symmetry between the A and B sublattices, similar to what happens in the creation of the edge state, generating the latticedistortion-induced effective gauge field $\mathbf{A}^{q}$. Eventually, the interaction of the incident laser beam with a graphene sheet subjected to lattice vibrations is given by the following equation by making the substitution

$$
\mathbf{p} \rightarrow \mathbf{p}+\mathbf{A}^{q}-e \mathbf{A} \text { in Eq.(1) }[5,6]
$$$$
\hat{H}=v_{\mathrm{F}} \sigma\left(\mathbf{p}+\mathbf{A}^{q}-e \mathbf{A}\right),
$$

where $\mathbf{A}$ is the electromagnetic gauge field of the incident laser beam. The electron-phonon interaction plays a key role in the Raman process. It is given by the deformation-induced gauge field $\mathbf{A}^{q}=\left(A_{x}^{q}, A_{y}^{q}\right)$, which can be expressed in terms of a change $\delta \gamma_{0}$ in the nearestneighbor transfer integral from the average value $\gamma_{0}$ $[5,20]$ as

$$
v_{\mathrm{F}} A_{x}^{q}=\delta \gamma_{0,1}-\frac{1}{2}\left(\delta \gamma_{0,2}+\delta \gamma_{0,3}\right), v_{\mathrm{F}} A_{y}^{q}=\frac{\sqrt{3}}{2}\left(\delta \gamma_{0,2}-\delta \gamma_{0,3}\right),
$$

where $\delta \gamma_{0,1}, \delta \gamma_{0,2}$, and $\delta \gamma_{0,3}$ are the changes in the transfer integrals for the $\pi$ bonds connected to three neighbors.

Using Eqs.(4) and (5), the optical transition is given by

$$
M^{\text {opt }}(\mathbf{A})=\left\langle\Psi_{\mathbf{k}}^{c}\left|-v_{\mathrm{F}} e \sigma \mathbf{A}\right| \Psi_{\mathbf{k}}^{v}\right\rangle,
$$

where $\Psi_{\mathbf{k}}^{c}$ and $\Psi_{\mathbf{k}}^{v}$ are the wave functions of the conduction and valence bands, respectively. The intensity of the Raman G-band, in which the longitudinal phonon mode is allowed owing to the intervalley transition at the armchair edge, is accordingly expressed as [6],

$$
\left|M^{\text {opt }}(\mathbf{A})\right|^{2} \propto \cos ^{2} \Theta \text {, }
$$

where $\Theta$ is the mutual angle between the armchair direction and the polarization direction of the incident laser beam. Figure 6 shows the atomic force microscopy (AFM) image and the Raman G-band observed in single layer armchair-edged graphene nanoribbons, whose typical size is $8 \mathrm{~nm}$ in width and $>1 \mu \mathrm{m}$ in length [21], together with the polarization angle dependence of the Gband intensity theoretically obtained. The polarization angle dependence of the intensity in the observation is in excellent agreement with that obtained from the theory, confirming the important contribution of electron wave interference.

\subsection{STM superlattice pattern and its fine} structure 
(a)

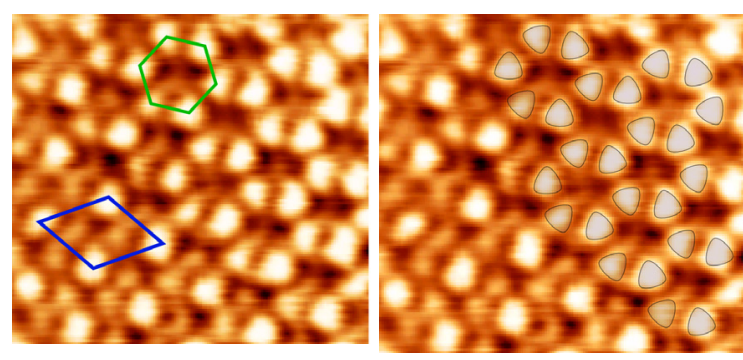

(c)

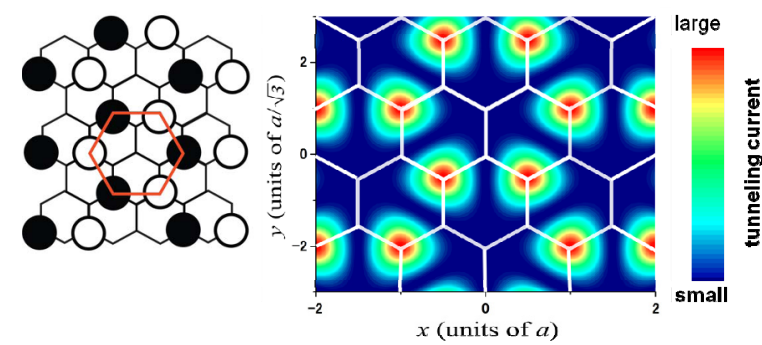

Fig. 7. Atomic-resolution STM lattice image $\left(1.4 \times 1.2 \mathrm{~nm}^{2}\right)$ $\mathrm{nm}^{2}$ ) taken armchair edge on the terrace near the step edge, which is approximately $1 \mathrm{~nm}$ away from the right end. Rhombus and hexagon indicate unit cell of the $\sqrt{3} \times \sqrt{3} R 30^{\circ}$ and honeycomb superlattice patterns, respectively. (b) Same to (a) with an overlaid three-fold symmetry pattern. The sample bias voltage is $20 \mathrm{mV}$. (c) $2 \mathrm{D}$ squared-amplitude mappings of the wave function near the armchair edge. Open and filled circles correspond to + and signs of the wave function phase, and their size corresponds to the amplitude of the wave function at each site. (d) Calculated 2D current mapping of the honeycomb superlattice pattern. Variation from dark blue to red represents increasing tunneling current (in arbitrary units). [Ref.13] (color)

Figure 7 shows the superlattice pattern observed near an armchair edge. In addition to the $(\sqrt{3} \times \sqrt{3}) R 30^{\circ}$ superlattice, a honeycomb superlattice pattern appears. Importantly, individual sites on the honeycomb superlattice have three-fold symmetric fine structure, as exhibited in Fig.7.(b). The origin of the honeycomb pattern appearing at an armchair edge can be understood in terms of electron wave interference in scattering events at the armchair edge in relation to intervalley transition ( $\mathrm{K} \leftrightarrow \mathrm{K}^{\prime}$ ). The wave function around the armchair edge is expressed by Eq.(2). Therefore, the spatial distribution of the local density of states (LDOS), which corresponds to the STM superlattice pattern, is given by the 2D squared-amplitude mapping of the wave function. The result of this mapping using Eq.(2) is shown in Fig.7(c), which reproduces well the experimentally observed honeycomb superlattice. Therefore, this confirms that intervalley scattering produces the honeycomb superlattice pattern at the armchair edge. The wave function's phase is important here. In the honeycomb superlattice, the signs of the wave function phase at adjacent sites are opposite to each other, which suggests that they have an antiphase relationship.
To understand the three-fold symmetrical fine structure observed in the honeycomb superlattice, it is necessary to calculate the spatial distribution of the current between the graphene and the STM tip [13,22]. The calculated current distribution is shown in Fig.7(d). The three-fold symmetrical fine structure observed in the STM atomic image is reproduced well in the calculated distribution. Taking into account the antiphase relationship between the wave functions at adjacent sites in the honeycomb superlattice, we conclude that the three-fold symmetry originates in antibonding coupling of the wave functions with a node in the middle between the two sites.

\section{Summary}

In graphene, the electronic structure of massless Dirac fermion moving on the hexagonal bipartite lattice is modified seriously by the presence of edges depending on the edge chirality. In the zigzag edge, broken symmetry of the pseudo-spin gives rise to the creation spinpolarized nonbonding edge state. In the armchair edge, electron wave interference takes place as a consequence of the participation of the K-K' intervalley transition. These geometrical dependence in the modifications can also be understood in terms of Clar's aromatic sextet rule in chemistry. In this paper we reviewed the phenomena appearing in STM/STS observations and Raman spectra. STM/STS observations of graphene edges hydrogen terminated reveal the presence of edge state in the zigzag edges. The Raman G-band as a function of the polarization direction of the incident laser beam proves the interference effect in the armchair edges. The formation of a honeycomb superlattice pattern with threefold symmetry fine structure observed in the vicinity of armchair edges is understood on the basis of the electron wave interference.

The present work is supported by the Grant-in-Aid for Scientific Research No. 20001006 from the Ministry of Education, Culture, Sports, Science and Technology, Japan.

\section{References}

1. N. Tajima, S. Sugawara, M. Tamura, R. Kato, Y. Nishio and K. Kajita, Eur. Phys. Lett. 80, 47002 (2007).

2. K. S. Novoselov, A. K. Geim, S. V. Morozov, D. Jiang, Y. Zhang, S. V. Dubonos, I. V. Grigorieva, and A. A. Firsov, Science 306, 666 (2004).

3. Y. Zhang, Y-W. Tan, H. L. Stormer, and P. Kim, Nature 438, 201 (2005).

4. A. H. Castro Neto, F. Guinea N. M. R. Peres K. S. Novoselov, and A. K. Geim, Rev. Mod. Phys. 81, 109 (2009).

5. K. Sasaki and R. Saito, Prog. Theor. Phys. Suppl. 176, 253 (2008).

6. K. Sasaki, R. Saito, K. Wakabayashi, and T. Enoki, J. Phys. Soc. Jpn. 79, 044603 (2010). 
7. S. E. Stein and R. L. Brown, J. Amer. Chem. Soc. 109, 3721 (1987).

8. M. Fujita, K. Wakabayashi, K. Nakada, and K. Kusakabe, J. Phys. Soc. Jpn. 65, 1920 (1996).

9. K. Nakada, M. Fujita, G. Dresselhaus, and M. S. Dresselhaus, Phys. Rev. B 54, 17954 (1996).

10. Y. Kobayashi, K. Fukui, T. Enoki, K. Kusakabe, and Y. Kaburagi, Phys. Rev. B 71, 193406 (2005).

11. Y. Kobayashi, K. Fukui, T. Enoki, and K. Kusakabe, Phys. Rev. B 73, 125415 (2006).

12. T . Enoki, Y. Kobayashi, and K. Fukui, Inter. Rev. Phys. Chem. 26, 609 (2007).

13. K. Sakai, K. Takai, K. Fukui, T. Nakanishi, and T. Enoki, Phys. Rev. B 81, 235417 (2010).

14. E. Clar, The Aromatic Sextet (Wiley, London, 1972).

15. K. Nakasuji and T. Kubo, Bull. Chem. Soc. Jpn. 77, 1791 (2004).

16. T. Wassmann, A. P. Seitsonen, A. Marco Saitta, M. Lazzeri, and F. Mauri, J. Amer. Chem. Soc. 132, 3440 (2010).

17. E. Lieb, Phys. Rev. Lett. 62, 1201 (1989).

18. G. U. Sumanasekera, G. Chen, K. Takai, J. Joly, N. Kobayashi, T. Enoki, and P. C. Eklund, J. Phys.: Condens. Matter. 22, 334208 (2010).

19. T. Enoki, M. Suzuki, and M. Endo, Graphite Intercalation Compounds and Applications (Oxford University Press, Oxford, 2003).

20. T. Kelly, Physics of Graphite (Applied Science Publishers, London, 1981).

21. L. G. Cançado, M. A. Pimenta, B. R. A. Neves, G. Medeiros-Ribeiro, T. Enoki, Y. Kobayashi, K. Takai, K. Fukui, M. S. Dresselhaus, R. Saito, and A. Jorio, Phys. Rev. Lett. 93, 047403 (2004).

22. T. Nakanishi and T. Ando, J. Phys. Soc. Jpn. 77, 024703 (2008). 